\title{
Viscosupplementation for Management of Knee Osteoarthritis from an Indian Perspective: An Expert Consensus Report
}

\author{
Sachin Tapasvi - Shubhranshu S. Mohanty - Kiran Kumar Vedavyasa Acharya • \\ Kanchan Bhattacharya $\cdot$ Raju Easwaran $\cdot$ Sujeet Narayan Charugulla
}

Received: May 22, 2019 / Published online: July 15, 2019

(C) The Author(s) 2019

\begin{abstract}
Introduction: Knee osteoarthritis (OA) is a progressive degenerative condition and is a significant contributor toward physical disability in the aging population. The current

Enhanced Digital Features To view enhanced digital features for this article go to https://doi.org/10.6084/ m9.figshare.8791010.

Electronic Supplementary Material The online version of this article (https://doi.org/10.1007/s40122019-0131-3) contains supplementary material, which is available to authorized users.
\end{abstract}

S. Tapasvi

The Orthopaedic Speciality Clinic, Pune, India

S. S. Mohanty

Department of Orthopaedics, King Edward

Memorial Hospital, Jaslok, Shushrusha and

Nanavati Hospitals, Mumbai, India

K. K. Vedavyasa Acharya

KMC Manipal, Manipal University, Manipal, India

K. Bhattacharya

Department of Orthopaedics, AMRI Hospital, Salt

Lake, Kolkata, India

R. Easwaran

Department of Orthopaedics, Shree Meenakshi Orthopedics and Sports Medicine Clinic, Delhi, India

S. N. Charugulla $(\square)$

Medical Affairs GG India, Dr. Reddy's Laboratories

Ltd, Hyderabad, India

e-mail: sujeetnc@drreddys.com treatment modalities for this condition focus on joint preservation with alleviation of symptoms. Intra-articular hyaluronic acid (IAHA) injections have emerged as the promising mainstay of nonsurgical treatment of $\mathrm{OA}$, especially in patients with mild-to-moderate OA and in certain subgroups of severe OA with comorbidities or with poor response to first-line therapy. The absence of standard guidelines or recommendations for the use of IAHA in India has led to vast variations in the usage of IAHA among practitioners. Hence, this consensusbased document aims to address the issue and establish simplified and easily implemented recommendations on the use of IAHA.

Methods: A group of 78 expert orthopedic surgeons discussed in detail the evidence on appropriate criteria for diagnosis, patient selection, and follow-up evaluation for knee OA at two national meetings. In subsequently held regional meetings, key discussion points and clinical experience-based answers were translated into a questionnaire to develop the final expert consensus-based statements for the use of IAHA in patients with knee OA.

Results: Various consensus statements were obtained on the basis of scientific evidence obtained from PubMed, Cochrane-indexed database, and guidelines related to viscosupplementation and knee $\mathrm{OA}$ as well as the experts' clinical experience. This document was drafted, reviewed, validated, and modified by 
the expert panel until a final agreement was reached.

Conclusion: In this pioneering attempt, the document lays down structured, expert consensus-based statements to guide and align practitioners on the appropriate use of IAHA in the Indian setting.

Funding: Dr. Reddy's Laboratories Ltd.

Keywords: Cartilage lesions; Comorbidities; Intra-articular hyaluronic acid; Kellgren and Lawrence grades; Oxford Knee Score; Recommendations

\section{INTRODUCTION}

Osteoarthritis (OA) is a degenerative joint disorder and is a leading cause of disability in the adult population [1]. Globally, OA is the eighth leading cause of physical impairment, with the knee being the most commonly affected joint [2]. In India, $\mathrm{OA}$ is the second most common rheumatological disease, with a prevalence rate ranging from $22 \%$ to $39 \%$ [3]. According to reports from the Planning Commission on Disease Burden (2011), OA accounted for half of all chronic conditions in the aged population ( $\geq 65$ years) [4]. Despite the mounting burden of this crippling condition, there is no established disease-modifying treatment available to date [5]. Furthermore, most of the pharmacological therapies for the management of OA are palliative in nature and have an unflattering risk-benefit ratio [5, 6]. Moreover, pharmacological rotation along with trial and error and the critical need for additional OA treatment have resulted in much dissatisfaction among the majority of patients [7]. Although the total knee replacement (TKR) surgical approach can effectively treat the pain, nonoperative treatment approaches aid in delaying the need for surgical interventions among patients who are not ideal candidates for surgery. In recent years, a nonsurgical measure, i.e., viscosupplementation, or intra-articular hyaluronic acid (IAHA) treatment, has gained popularity as a promising treatment modality in the management of knee OA. However, there is a dearth of robust evidence supporting the effectiveness, safety, and ideal candidate for intra-articular injections among the Indian population. Considering all the above points, there is a pressing need to evolve a consensus for IAHA use in the country.

The present document is a first-of-its-kind attempt to present consensus-based expert statements that can guide practitioners on IAHA use. These expert consensus-based statements are intended to be used along with the practitioner's judgment in order to optimize and individualize the treatment for the patient. This document further sets forth key points in evolving India-specific guidelines for consistent IAHA use by the medical fraternity for achieving effective management of knee OA.

\section{METHODS}

A group of 78 expert orthopedic surgeons participated, analyzed evidence, and discussed the unmet needs and role of IAHA in the management of knee $\mathrm{OA}$, in Indian settings at two national and six regional advisory board meetings, respectively. At the national meeting, the experts reviewed the available evidence on IAHA obtained through a literature search of MEDLINE (via PubMed), Cochrane-indexed databases, and guidelines on viscosupplementation. The experts provided their individual insights based on their clinical practice experience in the management of knee OA using IAHA and charted out key opinions as a part of initial inputs. Topics discussed by the experts to arrive at a consensus on the standardization of IAHA use in knee OA patients included evaluation, patient profile, indications, contraindications, and follow-up of patients treated with IAHA. These formed the basis of discussion at the second national advisory board meeting. Modified Delphi methodology was applied to achieve consensus on the initial inputs from the experts. Following the initial inputs, six regional meetings were convened across India. Key panel member experts from the national advisory board meeting presided over the discussion at their regional centers. During the regional meetings, the participating experts were provided with a questionnaire and their opinions were collated on demographics, pain and 
functional assessment, key imaging features, patient profile for IAHA, alternate treatment options for knee OA, follow-up evaluation of knee OA, and challenges in the management of knee OA with IAHA.

This article is based on previously conducted studies and does not contain any studies with human participants or animals performed by any of the authors.

\section{RESULTS}

The discussion points obtained from the experts, based on the scientific evidence discussion and their clinical practice judgment, were considered as expert consensus-based statements. A summary of clinical statements obtained on various topics have been discussed below.

\section{Why Is the Diagnosis of Knee OA Crucial?}

The diagnostic evaluation of suspected knee OA is crucial to determine an appropriate treatment strategy. The various elements of diagnostic evaluation include patient history, physical examination, and imaging studies [8]. Several subjective measures, including patient-reported questionnaires, are also used to assess the symptoms and evaluate the functions of the knee [9]. The primary focus of the clinical examination of the knee is to elicit any abnormalities related to the following [10]:

- Status of the articular cartilage

- Affection of the patellofemoral joint

- Status of synovial tissue

Plain radiographs form an important diagnostic imaging modality in the evaluation of knee OA [8]. Important weight-bearing views include weight-bearing anteroposterior (AP) and lateral views and Rosenberg view [11]. The Kellgren and Lawrence system of staging of knee $\mathrm{OA}$ is used to grade the severity of the disease and is based on radiographic findings $[12,13]$. Magnetic resonance imaging (MRI) is used for the demonstration of cartilage status, which cannot be detected on plain radiographs [1]. Distinguishing noninflammatory arthritis (knee OA) from inflammatory arthritis (rheumatoid arthritis, RA) is important in arriving at a proper diagnosis, since the treatment plan for either differs significantly. Important distinguishing features include:

- Pain tends to worsen as the day progresses in noninflammatory arthritis, whereas pain is severe during the morning hours in inflammatory arthritis [8].

- Stiffness does not last for more than half an hour in noninflammatory arthritis, whereas stiffness lasts for more than half an hour in inflammatory arthritis [14].

- Contrary to inflammatory arthritis, symptoms such as pain, stiffness, reduced range of movements, swelling, and crepitus are not associated with systemic features in noninflammatory arthritis [15].

Clinical recommendations obtained from the 78 experts for the diagnosis of knee $\mathrm{OA}$ are summarized in Table 1 . The experts acknowledged that distinguishing knee OA (noninflammatory form of arthritis) from rheumatoid arthritis (inflammatory form of arthritis) is crucial to implement appropriate treatment. They further suggested that the American College of Rheumatology criteria can be used clinically in the diagnosis of knee OA.

\section{What are the Minimally Invasive Interventional Options Available for the Symptomatic Management of Osteoarthritic Chronic Knee Pain?}

1. Intra-articular Injections

(a) Corticosteroids: Intra-articular corticosteroid injections have been traditionally used in treatment of knee OA patients who fail to respond to conservative treatments. These injections are preferred in patients who have persistent pain and have failed to alter modifiable risk factors, including weight and activity level. Methylprednisolone acetate, triamcinolone acetonide, and triamcinolone hexacetonide are commonly used intra-articular corticosteroid 
Table 1 Overview of expert consensus-based statements on diagnosis and various imaging techniques for knee osteoarthritis $(\mathrm{OA})$

\section{Clinical statements}

Pain level and functional Clinical assessment of pain in the outpatient department and pain assessment questionnaires disability should be considered

Patients should be assessed for the presence of any pain at rest or during sleep and should be questioned about the need for analgesics and walking aids for carrying out routine activities

Oxford Knee Score can be considered as the easiest to use and is a patient-acceptable, painscoring questionnaire

Visual analog scale (VAS) and Western Ontario and McMaster Universities Osteoarthritis Index (WOMAC) scores are other pain scores for patients with knee OA

Assessment of activities of daily living is an important tool in the assessment of pain evaluation in patients with knee $\mathrm{OA}$

The most commonly used questions used to assess limitations include the ability to perform daily routine activities; the need for analgesics to relieve pain; and any limitations or pain during walking, climbing, and squatting on the floor or getting up from the sitting position

In a suspected case of knee OA, the clinical examination should be detailed

Clinical examination

Diagnose the condition with reasonable accuracy, especially when the role of arthroscopy in the management of knee $\mathrm{OA}$ itself is debatable

Apart from the routine knee examination, assessment for the presence of patellofemoral crepitus to identify patellofemoral arthritis should be considered during the clinical examination

In the examination of grade $3 \mathrm{OA}$ associated with meniscal and chondral injuries, the McMurray test may not be helpful, as it may show false-positive results

Radiographic imaging Adequate radiographs of the knee joint should be the preferred imaging tool for the diagnosis of knee OA

The weight-bearing anteroposterior (AP) view and lateral view of the knee joint should be taken in all patients during evaluation

Radiographs of the knee joint should always be taken in the standing position

Standing AP scanogram of both lower limbs in a patient with knee OA is important to assess extra-articular deformity in the setting of previous trauma and tibia vara, which is common in India

The important radiological features for the diagnosis of knee OA include reduction in joint space, osteophyte formation, malalignment, and Kellgren and Lawrence $(\mathrm{KL})$ grading criteria 
Table 1 continued

Clinical statements
$\begin{aligned} & \text { Magnetic resonance } \\ & \text { imaging }\end{aligned}$
whom OA is suspected
MRI should not be advised in all cases indicated for intra-articular hyaluronic acid (IAHA).
This imaging tool should be advised only when there is a dilemma in the diagnosis of knee
OA, e.g., when an association between ligamentous laxity, AP instability, medial
patellofemoral ligament involvement, significant meniscal tears, and isolated cartilage lesions
is suspected
In any young patient presenting with knee pain because of sport or nonsport injury and not
relieved of pain even after 3 weeks of conservative treatment, cartilage injury should be
suspected and MRI performed
T2 mapping is not performed routinely in clinical practice; it is considered only when cartilage
lesions are suspected
T2 mapping of MRI helps in the assessment of cartilage lesions and meniscal damage
MRI is indicated when pain and other symptoms outweigh the radiological findings

injections. Evidence suggests that longterm injections of glucocorticoids lead to greater structural damage and loss of cartilage volume. Also, risk of postinjection flare-up occurring a few hours after the injection and lasting for 2-3 days is noted with intra-articular corticosteroid injections [16].

(b) Viscosupplementation: Intra-articular hyaluronic acid injections are indicated in patients with symptomatic OA who have not responded to firstline therapy and conservative management. Hyaluronic acid (HA) enhances the viscoelastic properties of the synovial fluid, increases the production of endogenous HA, and supports the chondrocyte matrix from degradation [16].

(c) Platelet-rich plasma (PRP): Platelet-rich plasma is an autologous blood product comprising concentrated platelets above the baseline blood value found in healthy individuals. Platelets contain a host of growth factors that promote regeneration of bone and degenerated cartilage in the knee. Clinical studies have shown that PRP improves function and quality of life and decreases OA pain [16].

2. Radiofrequency Therapy (RF)

Radiofrequency therapy is an emerging therapeutic option for patients who are not fit for surgery and for patients with persistent, chronic pain following knee surgery. Cooled RF is a novel therapeutic approach used in the management of chronic osteoarthritic knee pain. In this technique, a coolant is allowed to continuously circulate internally at the electrode probe tip, thereby enabling greater energy delivery and creation of larger lesions for denervation. In a survey, cooled RF demonstrated greater than $80 \%$ pain relief compared to diagnostic nerve blocks in 33 patients with knee OA [16]. However, the long-term outcomes are not yet available for this treatment modality. Although 
significant analgesia is achieved with RF ablation therapy, clinicians have limited its usage to patients refractory to conservative treatment because of its invasive nature. Many studies have also implicated diagnostic genicular nerve block to ensure correct nerve identification.

3. Extracorporeal Shockwave Therapy (ESWT) This is a novel approach used in the treatment of knee OA. Evidence suggests that in early- to mid-stage knee OA patients with MRI findings of bone marrow edema (BME), ESWT effectively reduced pain within 3 months and demonstrated radiographic evidence of decreased BME within 3 months [16].

\section{What are the Indications for Viscosupplementation?}

Intra-articular injections of HA are considered in the following patients:

- Patients with symptomatic Kellgren and Lawrence (KL) grade 2 and 3 knee OA (Table 2) [12, 17]

- Patients with contraindications or intolerance to nonsteroidal anti-inflammatory drugs (NSAIDs) [18]

- Patients with KL grade 4 knee OA not willing to undergo or not determined to postpone surgery (Table 2) [12, 19]

- Chondrocalcinosis [18]

- Sports persons with any cartilage injury [20]

Clinical evidence supports the use of IAHA in patients intolerant to nonsteroidal anti- inflammatory drugs (NSAIDs) and patients unwilling to undergo or wishing to defer surgery. In a meta-analysis that included five trials and 712 patients with symptomatic knee OA, more gastrointestinal tract adverse events were noted in patients treated with NSAIDs compared to those treated with IAHA [21]. In a retrospective analysis, Altman et al. reported that the median time to TKR was delayed by more than 1 year in patients receiving one dose of HA injection, whereas it was 0.3 years in patients not receiving $\mathrm{HA}$ injections. In patients who received five or more HA injections, TKR was delayed by 3.6 years [19]. In a retrospective longitudinal study involving 14,782 knee OA patients, effectiveness of HA injections in delaying TKR surgery was assessed. In the study, bivariate analysis conducted on 1162 patients with total knee arthroplasty showed that the mean time from diagnosis to TKR was significantly higher in the HA group than in the nonHA group (864 vs. 573 days, respectively; $p<0.0001)$ [22].

In a critical literature review analysis by Maheu et al., IAHA was found to provide a moderate symptomatic benefit to knee OA patients without safety concerns. The analysis further highlighted that the benefit-to-risk ratio offered by viscosupplementation is good among the pharmacologic options for knee OA. It can lead to overall gain in quality-adjusted life years and a significant delay in time to TKR in a patient [23].

Furthermore, in a real-life setting, good evidence on the efficacy of IAHA when compared with IA corticosteroids is available. A repeated course of IAHA in a real-world setting has

Table 2 Kellgren and Lawrence classification scale for knee osteoarthritis severity [12]

\begin{tabular}{ll}
\hline Grade & Description \\
\hline 0 & No radiographic features of osteoarthritis \\
1 & Formation of osteophytes on the tibial spines \\
2 & Presence of periarticular ossicles \\
3 & Narrowing of joint cartilage associated with sclerosis of subchondral bone \\
4 & Small pseudocystic areas with sclerotic walls situated in the subchondral bone \\
\hline
\end{tabular}


demonstrated an improvement in pain or joint function lasting up to 40 months along with reduction in the concomitant analgesic usage to half. The clinical benefit of IAHA on knee OA translates in the form of allowing lubrication and shock absorption in the joint and the reestablishment of joint homeostasis through induction of endogenous HA production [24].

Clinical recommendations obtained from the experts for indication for viscosupplementation are summarized in Table 3.

\section{What are the Indications for Viscosupplementation in Knee OA Post Arthroscopic Surgery?}

Arthroscopic surgery is the preferred treatment option in patients with knee OA who fail to respond to conservative treatment approaches and in whom a symptomatic meniscal lesion is suspected. The primary goal of arthroscopic surgery is to stabilize the torn meniscus and/or chondral lesion and remove the products of cartilage wear and inflammatory cells from the joint space to counter the onset of painful inflammatory phases. Arthroscopic surgery is predominantly indicated to tackle meniscal tears, isolated cartilage lesions, impinging osteophytes, and loose bodies [25].

The following are important considerations for IAHA instillation following arthroscopic surgery:

- Patients with persistent knee pain following arthroscopy [26]

- Patients with mild knee OA who have undergone arthroscopic partial meniscectomy and debridement [27]

In a prospective study, the short- and longterm effects of post arthroscopic IAHA were evaluated in 80 patients with persistent knee pain. Of the 80 patients, 40 underwent arthroscopic knee joint lavage and debridement (group A), whereas the rest of the patients underwent the same procedure with additional IAHA injections (group A + HA). The study revealed that more than one-third $(39.5 \%)$ of patients continued to complain of pain while walking after 1 year. However, in group 2 $(\mathrm{A}+\mathrm{HA})$, a steady increase in the proportion of pain-free patients was noted; only $13.9 \%$ of the

Table 3 Overview of expert consensus-based statements on indications for viscosupplementation

\section{Clinical statements}

Viscosupplementation Those with symptomatic Kellgren and Lawrence (KL) grades 1 and 2 knee osteoarthritis (OA) could be considered ideal patients for intra-articular hyaluronic acid (IAHA)

In patients with $\mathrm{KL}$ grade $3 \mathrm{knee} \mathrm{OA}$ who are unwilling to undergo surgery, IAHA is an alternative option

In elderly patients who have bilateral knee OA and are willing to undergo surgery, IAHA could be considered as a treatment option for the knee that is affected

IAHA injections can be considered an extended indication in patients with KL grades 3 and 4, and counseling plays a key role in influencing the compliance of these patients

Young patients with KL grade 2 and 3 knee OA who have failed to respond to a conservative line of treatment can be treated using IAHA

Patients who are unwilling to undergo or wish to defer surgery can be considered for IAHA. However, these patients should be made aware that IAHA is just an alternative treatment option

Patients presenting with sports injuries associated with minimal chondral lesions and those with chondropenia can undergo treatment with IAHA 
patients complained of pain while walking at the end of 1 year (Fig. 1) [25].

Clinical recommendations obtained from the experts for indication for viscosupplementation in patients with arthroscopic surgery are summarized in Table 4.

\section{What are the Contraindications for Viscosupplementation?}

The following are the absolute contraindications for IAHA:

- Knee joint infections [28]

- Skin disease or infections in the area around the injection site [28]

- Inflammatory arthritis [18]

- Acute exacerbation of OA [18]

- Edema of the bone [18]

The following are the relative contraindications for IAHA:

- Patients with $>30 \mathrm{~kg} / \mathrm{m}^{2}$ body mass index [29]

- Patients with severe extremity malalignment [29]

Any intra-articular injection is associated with several local and systemic adverse effects. Injection-related pain, post-injection flare, fat atrophy, skin pigment changes, and joint infection are the common local adverse effects; systemic effects that may develop following intra-articular injections include disruption in blood glucose level control and blood pressure control, sepsis, inhibition of the hypothalamo-pituitary-adrenal axis, and death [30].

In line with the evidence and experts' recommendations, the contraindications of viscosupplementation are summarized in Table 5.

\section{What are the Follow-Up Evaluations for Patients Treated With IAHA?}

In the follow-up evaluation of patients with knee OA, several pain-scoring systems, including visual analog scale (VAS), Western Ontario and McMaster Universities Osteoarthritis Index (WOMAC), and Oxford Knee Score, are used. Compared with other scoring systems, the ease of use makes the Oxford Knee Score a useful tool for clinical use, both during evaluation and follow-up [9].

Experts acknowledged the importance of evaluating the patient post IAHA treatment and clinical recommendations arising from the discussion are summarized in Table 6.
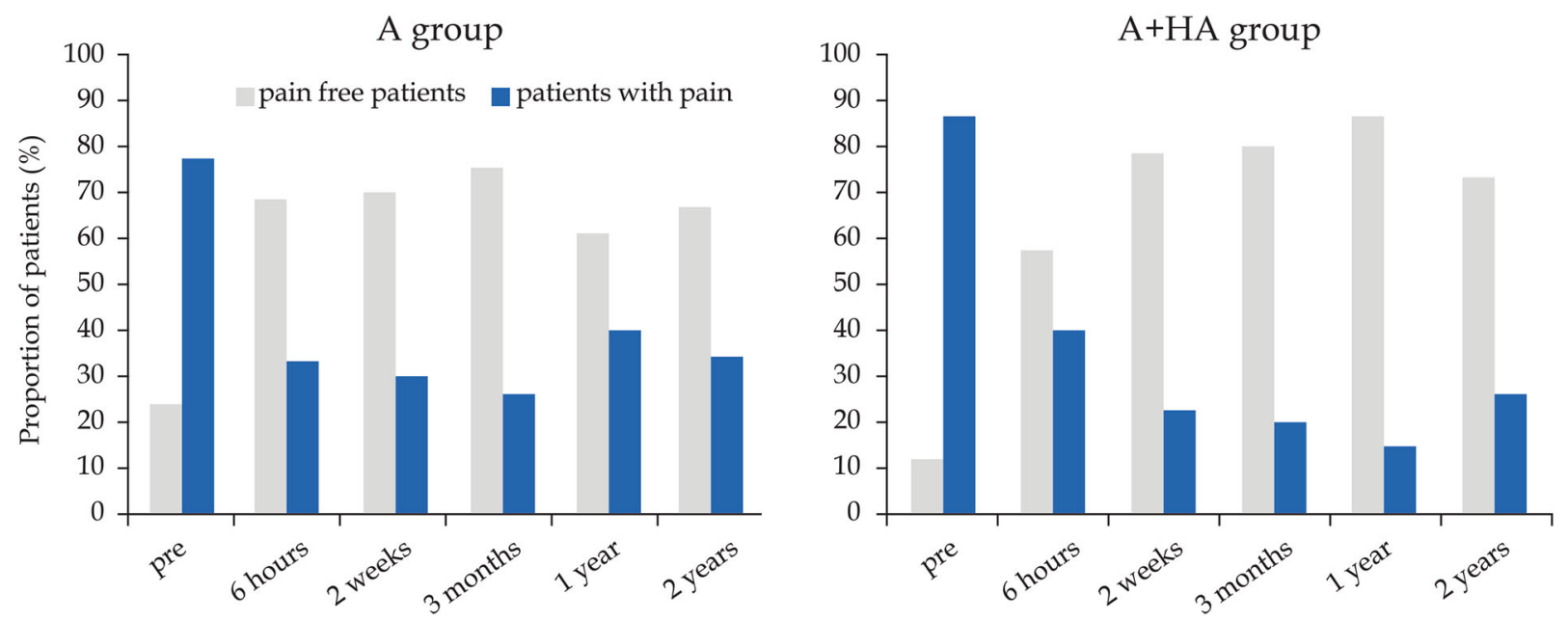

Fig. 1 Pain on walking $100 \mathrm{~m}$ in patients treated with arthroscopy (group A) and those treated with arthroscopy and HA (group A + HA) [25]. Grey bars represent pain-free patients, and blue bars represent patients with pain 
Table 4 Overview of expert consensus-based statements on indications for viscosupplementation in knee OA patients after arthroscopic surgery

\begin{tabular}{ll}
\hline Clinical statements \\
\hline $\begin{array}{l}\text { Viscosupplementation } \\
\text { indication }\end{array}$ & $\begin{array}{c}\text { Intra-articular hyaluronic acid (IAHA) is indicated in knee OA patients following arthroscopic } \\
\text { surgery for meniscal tears and isolated cartilage lesions } \\
\text { The administration of IAHA after arthroscopy helps reduce pain, improves mobility, and } \\
\text { increases activities of daily living. It is also chondroprotective in nature } \\
\text { The beneficial effects of arthroscopic surgery are prolonged with the use of IAHA in cases with } \\
\text { cartilage lesions detected during arthroscopy } \\
\text { Experts further added that the ideal time for the administration of IAHA following arthroscopy } \\
\text { is } 4-6 \text { weeks, as joint irritation and arthroscopy-related synovitis reduce and as joint function } \\
\text { improves by } 4 \text { weeks of the arthroscopic procedure }\end{array}$ \\
\hline
\end{tabular}

Table 5 Overview of expert consensus-based statements on contraindications of viscosupplementation

\begin{tabular}{l}
\hline Clinical statements \\
\hline $\begin{array}{l}\text { Kiscosupplementation } \\
\text { contraindications } \\
\text { the injection site are absolute contraindications for intra-articular hyaluronic acid } \\
\text { (IAHA) } \\
\text { In suspected cases of acute exacerbation of osteoarthritis, evaluation of C-reactive protein } \\
\text { (CRP) is essential and IAHA should not be given if CRP is high } \\
\text { Bone marrow edema, unless associated with cartilage damage, should be considered an } \\
\text { absolute contraindication for IAHA } \\
\text { Any surgical contraindication, such as uncontrolled diabetes mellitus, should be considered } \\
\text { as a contraindication for IAHA. One of the systemic adverse effects noted following } \\
\text { intra-articular injections is disruption in blood glucose levels. Therefore, we can consider } \\
\text { that IAHA should be contraindicated in patients with poor glycemic levels }\end{array}$ \\
\hline
\end{tabular}

Table 6 Overview of expert consensus-based statements on follow-up evaluations after viscosupplementation treatment

\section{Clinical statements}

Viscosupplementation followup evaluation
The Oxford Knee Score is the most beneficial tool used in the follow-up evaluation of patients with knee osteoarthritis treated with intra-articular hyaluronic acid

Other measures used in clinical practice for follow-up evaluation include visual analog scale, reduction in the use of analgesics, clinical radiograph of knee joint, assessment of range of movements, patient feedback, and assessment for improvement in the activity level, such as walking and climbing stairs 


\section{What are the Key Challenges in the Management of Knee OA With IAHA?}

From the clinicians' perspective, certain uncertainties and controversies, such as inconsistency between standard clinical guidelines and published literature, exist regarding the efficacy of IAHA in the management of knee OA [31]. Experts acknowledged that there is a dearth of evidence and there are no structured guidelines for this potentially beneficial knee OA modality and the key challenges identified by the experts are listed in Table 7.

\section{What are the Various Choices of Viscosupplements?}

Viscosupplements are chemically cross-linked HA molecules with a high molecular weight and long shelf-life. These cross-linked hyaluronans have increased elastoviscous properties compared with HAs. Exogenous HAs are administered in viscosupplementation therapy. Evidence suggests that HA preparations with higher molecular weight are associated with significant clinical outcomes when compared to those with lower molecular weight (Fig. 2) [32]. In an observational cohort study including 30,417 incident HA users, effectiveness of low molecular weight HA (LMWHA), moderate

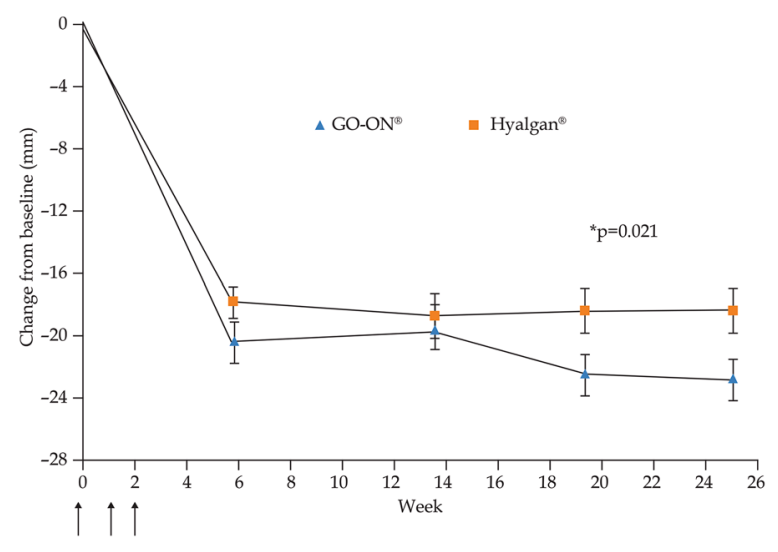

Fig. 2 Intermediate MW HA $\left(\mathrm{GO}-\mathrm{ON}^{\circledR}\right)$ demonstrating the trend for a better pattern of response throughout the study compared to low MWHA [32]

molecular weight HA (MMWHA), and high molecular weight HA (HMWHA), in prevention or delay of knee surgery in knee OA patients, was compared. The study reported no significant difference in likelihood of surgical interventions among users of LMWHA, MMWHA, and HMWHA [33].

Reports from a randomized trial indicate that cross-linked HA was not inferior to biologically fermented HA and was associated with an improved WOMAC pain score (Fig. 3) [34]. In a randomized, double-blind trial, patients treated with chemically cross-linked hyaluronan had

Table 7 Overview of expert consensus-based statements on key challenges of the viscosupplementation treatment

\begin{tabular}{|c|c|}
\hline & Clinical statements \\
\hline \multirow[t]{8}{*}{$\begin{array}{l}\text { Challenges with use of intra-articular hyaluronic } \\
\text { acid }\end{array}$} & $\begin{array}{l}\text { Counseling the patients regarding the number of injections and } \\
\text { prognosis }\end{array}$ \\
\hline & Timing of injection \\
\hline & Number of injections required \\
\hline & Selection of the right patient profile \\
\hline & Compliance and cost of injections \\
\hline & Follow-up evaluation \\
\hline & Longevity of the patient \\
\hline & Paucity of strong clinical evidence \\
\hline
\end{tabular}




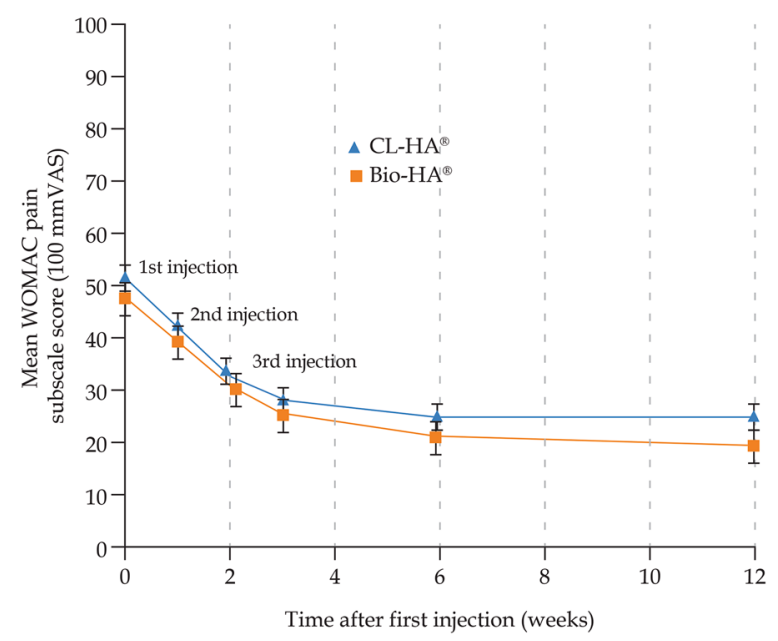

Fig. 3 Improved WOMAC score with cross-linked HA compared to biologically fermented HA [34]

improved pain scores compared to patients treated with avian-derived hyaluronan [35].

Hyaluronic acid preparations with prolonged residence time allow a reduced number of injections to achieve long-term efficacy in knee OA [19].

In line with the evidence, experts agreed that molecular weight is one of the key product characteristics to be considered while choosing an IAHA. The experts further acknowledged that in clinical practice, more than product characteristics, the efficacy, clinical evidence, and statutory body approval should be given importance. Table 8 summarizes the statements on the choice of viscosupplementation agent.

\section{What is the Injection Technique used for Viscosupplementation?}

It is important that IAHA be administered by an authorized physician or medical professionals in facilities well suited for intra-articular injections [36]. Experts recommended that the knee- extended and knee-flexed injection techniques can be used. Specific expert clinical recommendations pertinent to injection technique are presented in Table 9.

\section{What is the Safety and Efficacy of Long- Term Use of HA Injections Through Repeated Courses of Treatment in Patients With Knee OA?}

A systemic review of 17 articles (seven randomized controlled trials and 10 cohort studies) assessed the efficacy and safety of repeated treatment courses with IAHA, in terms of single reinjection cycle to four repeat injection cycles. Eleven studies assessed the effects of one reinjection of $\mathrm{HA}$ on reduction of pain in knee OA patients, five studies evaluated at least two repeated courses of IAHA, and one study evaluated the effects of either one or two repeated courses. All studies demonstrated pain reduction from baseline in the IAHA treatment group throughout the initial treatment cycle, and either sustained or further reduced pain throughout the repeated courses of treatment. The study with the longest follow-up repeated IAHA injection every 6 months for 25 months. Pain decreased after the first course and continued to decrease until the end of the study, with an approximate $55 \%$ reduction in pain compared to baseline [37].

\section{DISCUSSION}

There is a wide gap in the effective management of knee OA, specifically in patients who are unresponsive to conservative treatment and are unwilling to undergo or are unsuitable for surgical procedures. In such patients, minimally invasive adjuvant therapies, including intra-articular injections, radiofrequency therapy, and

Table 8 Overview of expert consensus-based statements on choice of viscosupplementation agents

Clinical statements

Viscosupplements Increased molecular activity and prolonged residence time increase the efficacy of the product and account for fewer injections

Stabilization of hyaluronic acid prevents oxidation and facilitates longer residence time 
Table 9 Overview of expert consensus-based statements on injection technique for viscosupplementation

\begin{tabular}{ll}
\hline Clinical statements \\
\hline Injection & Knowledge of the advantages and disadvantages of both techniques ensures a better clinical outcome \\
technique & The selection of technique depends on the surgeon's preference and comfort level \\
The procedure should be performed in a sterile environment, preferably in an operating theater \\
Following administration of intra-articular hyaluronic acid (IAHA), the knee joint should be taken \\
through a range of motions to facilitate even distribution of hyaluronic acid inside the joint and to \\
block the path of the injection \\
Following injection of IAHA, the patient should be advised to avoid sports activities for $24 \mathrm{~h}$ \\
In patients with severe patellofemoral joint issues and in later stages of the disease, IAHA should not be \\
injected in the knee-extended position \\
In patients with flexion deformity, the para-tendinous approach is more beneficial \\
Injection into the fat pad should be avoided, as it worsens the pain
\end{tabular}

extracorporeal shockwave therapy, have emerged as promising options. Unraveling the hidden strength of these novel approaches is crucial to improve the quality of life of patients with symptomatic knee OA.

Extensive critical literature reviews of 17 meta-analyses of randomized controlled trials have reported that IAHA provides moderate symptomatic benefit to knee OA patients without any major safety concerns. A systematic clinical evidence review of 11 clinical studies reported that a single injection of non-animal stabilized hyaluronic acid (NASHA) demonstrated sustained and effective relief of knee OA symptoms and was associated with a low rate of adverse events [38].

There is a gradual demographic shift in knee OA to the younger population because of repeated trauma to the cartilage. Younger individuals engaging in sports activities involving repeated chronic stress were considered to be at an increased risk of degeneration of the knee joint and consequent development of knee OA. Additionally, experts acknowledged the rise in patellofemoral arthritis among younger individuals with desk jobs and sitting for prolonged periods.

Experts suggested that distinguishing cases indicated for arthroscopy from those indicated for IAHA therapy was important in view of the further treatment course. Differences in opinion were evident in relation to the concomitant use of steroids and IAHA in patients with knee OA. In patients with knee OA with significant knee effusion, knee aspiration injection of steroid followed by IAHA was more effective. In patients with OA with disease progression, the natural concentration and distribution of HA, an integral component of synovial fluid, diminish, thereby resulting in the degradation of viscoelastic properties. In a prospective study, which included 80 patients with persistent knee pain, instillation of IAHA following arthroscopy for chondral lesions and meniscal tears resulted in significant reduction in knee pain. The experts opined that IAHA injections can be considered a beneficial nonsurgical treatment, as these restore the HA concentration through stimulated endogenous HA production.

Clinical evidence suggests that HA preparations that are derived from rooster comb tissue have a short residence time in the joint; therefore, multiple injections are required to achieve efficacy. However, NASHA, which is synthesized by streptococci, has a prolonged residence time in the joint. This could be attributed to the stabilization process, which creates a viscous gel with increased density, retaining its 
biocompatibility. As NASHA closely resembles the body's own HA, it provides a long-lasting effect. Furthermore, guidelines too have recommended the use of IAHA in knee OA. The 2013 American Academy of Orthopaedic Surgeons guidelines for knee OA, which strongly recommended against the use of IAHA in patients with knee $\mathrm{OA}$, changed their stance in 2014, suggesting that IAHA could be used as a supplementary tool in knee OA, based on the surgeon's clinical discretion [39].

\section{CONCLUSION}

Intra-articular hyaluronic acid injections constitute one of the minimally invasive therapeutic options available in the management of knee OA.

As a result of the lack of standard clinical practice recommendations on IAHA in India, patient profile for IAHA and appropriate time for IAHA are still areas of ambiguity among practitioners. The clear consensus-based expert statements on various aspects related to the use of IAHA in patients of knee OA will not only empower practicing clinician's decision-making for the right patient for IAHA but also help in the consistent use of IAHA in the country. To date, there has not been a published consensus statement for usage of $\mathrm{HA}$ to treat chronic bilateral knee pain secondary to $\mathrm{OA}$ in India to the best of our knowledge. Thus, the authors representing senior orthopedic surgeons have attempted to do so. During the consensus meeting, use of IAHA in treatment of other joints was not discussed, as the consensus was specific to knee OA pathology.

In our pioneering attempt, we firmly believe that this document shall further lay the foundation for developing specific guidelines on the management of knee OA using IAHA, across various settings in India.

\section{ACKNOWLEDGEMENTS}

We would like to thank all the national and regional advisory board panel experts for their active participation in the development of this consensus document.

Funding. This project was funded by Dr. Reddy's Laboratories Ltd. Funding was provided for arranging the meeting, facilitating the development of this manuscript and the journal's Rapid Service Fee. All authors had full access to the articles reviewed in this manuscript and take complete responsibility for the integrity and accuracy of this manuscript.

Editorial Assistance. Editorial assistance in the preparation of this article was provided by Dr. Kavitha Ganesha of BioQuest Solutions Pvt Ltd. Support for this assistance was funded by Dr. Reddy's Laboratories Ltd.

Authorship. All named authors meet the International Committee of Medical Journal Editors (ICMJE) criteria for authorship for this article, take responsibility for the integrity of the work as a whole, and have given their approval for this version to be published.

Disclosures. Sachin Tapasvi is on the scientific advisory board for Dr Reddy's Laboratories and has received educational grant for planning and preparing this document. Shubhranshu S. Mohanty is on the scientific advisory board for Dr Reddy's Laboratories and has received educational grant for planning and preparing this document. Kiran Kumar Vedavyasa Acharya is on the scientific advisory board for Dr Reddy's Laboratories and has received educational grant for planning and preparing this document. Kanchan Bhattacharya is on the scientific advisory board for Dr Reddy's Laboratories and has received educational grant for planning and preparing this document. Raju Easwaran is on the scientific advisory board for Dr Reddy's Laboratories and has received educational grant for planning and preparing this document. Sujeet Narayan Charugulla is an employee of Dr. Reddy's Laboratories Ltd.

Compliance with Ethics Guidelines. This article is based on previously conducted studies and does not contain any studies with human 
participants or animals performed by any of the authors.

Data Availability. Data sharing is not applicable to this article as no datasets were generated or analyzed during the current study.

Open Access. This article is distributed under the terms of the Creative Commons Attribution-NonCommercial 4.0 International License (http://creativecommons.org/licenses/ by-nc/4.0/), which permits any noncommercial use, distribution, and reproduction in any medium, provided you give appropriate credit to the original author(s) and the source, provide a link to the Creative Commons license, and indicate if changes were made.

\section{REFERENCES}

1. Furtado C, Deshpande M, D'mello Z, et al. Clinical profile of patients undergoing total knee replacement (TKR)-case based series. IOSR J Dental Med Sci. 2016;15:95-101.

2. Mathers CD, Bernard C, Iburg KM, et al. Global programme on evidence for health policy. Discussion paper no. 54. 2003. http://www.who.int/ healthinfo/paper54.pdf. Accessed 06 June 2019.

3. Pal CP, Singh P, Chaturvedi S, et al. Epidemiology of knee osteoarthritis in India and related factors. Indian J Orthop. 2016;50:518-22.

4. Ministry of Health and Family Welfare. Working Group on Disease Burden: non-communicable diseases. 2011. http://planningcommission.nic.in/ aboutus/committee/wrkgrp12/health/WG_3_ 2non_communicable.pdf. Accessed 06 June 2019.

5. Upadhyay S, Varma HS, Chaurasia S, et al. Randomized blinded comparative analysis of autologous mononuclear medullary fraction and viscosupplementation in grade 0 and grade 1 radiographic scale of knee osteoarthritis. Indian J Pain. 2014;28:134-42.

6. Crichton B, Green M. GP and patient perspectives on treatment with non-steroidal anti-inflammatory drugs for the treatment of pain in osteoarthritis. Curr Med Res Opin. 2002;18:92-6.

7. Zhao SZ, Wentworth C, Burke TA, et al. Drug switching patterns among patients with rheumatoid arthritis and osteoarthritis using COX-2 specific inhibitors and non-specific NSAIDs. Pharmacoepidemiol Drug Saf. 2004;13:277-87.

8. Michael JW, Schlüter-Brust KU, Eysel P. The epidemiology, etiology, diagnosis, and treatment of osteoarthritis of the knee. Dtsch Arztebl Int. 2010;107:152-62.

9. Collins NJ, Misra D, Felson DT, et al. Measures of knee function. Arthritis Care Res (Hoboken). 2011;63:S208-28.

10. Rossi R, Dettoni F, Bruzzone $\mathrm{M}$, et al. Clinical examination of the knee: know your tools for diagnosis of knee injuries. Sports Med Arthrosc Rehabil Ther Technol. 2011;3:25.

11. Saadat E, Link TM. Current radiographic diagnosis for osteoarthritis of the knee. In: Majumdar S, editor. Advances in MRI of the knee for osteoarthritis. Singapore: World Scientific; 2010. p. 69-71.

12. Kellgren JH, Lawrence JS. Radiological assessment of osteo-arthrosis. Ann Rheum Dis. 1957;16: 494-502.

13. Hayashi D, Roemer FW, Jarraya M, et al. Imaging of osteoarthritis. In: Guglielmi G, editor. Geriatric imaging. New Jersey: Springer; 2013. p. 97.

14. Davis JM III, Moder KG, Hunder GG. History and physical examination of the musculoskeletal system. In: Firestein GS, editor. Kelly's textbook of rheumatology. 9th ed. Philadelphia: Elsevier Health Sciences; 2012. p. 560.

15. Hunter DJ, Felson DT. Osteoarthritis. BMJ. 2006;332:639-42.

16. Urits I, Patel R, Adamian L, et al. Minimally invasive interventional management of chronic knee pain. J Knee Surg. 2018;32(1):72-9.

17. Navarro-Sarabia F, Coronel P, Collantes E, et al. A 40-month multicentre, randomised placebo-controlled study to assess the efficacy and carry-over effect of repeated intra-articular injections of hyaluronic acid in knee osteoarthritis: the AMELIA project. Ann Rheum Dis. 2011;70:1957-62.

18. Legré-Boyer V. Viscosupplementation: techniques, indications, results. Orthop Traumatol Surg Res. 2015;101(1 Suppl):S101-8.

19. Altman R, Lim S, Steen RG, et al. Hyaluronic acid injections are associated with delay of total knee replacement surgery in patients with knee osteoarthritis: evidence from a large U.S. health claims database. PLoS One. 2015;10(12):e0145776. 
20. McAdams TR, Mithoefer K, Scopp JM, et al. Articular cartilage injury in athletes. Cartilage. 2010;1: 165-79.

21. Bannuru RR, Vaysbrot EE, Sullivan MC, et al. Relative efficacy of hyaluronic acid in comparison with NSAIDs for knee osteoarthritis: a systematic review and meta-analysis. Semin Arthritis Rheum. 2014;43:593-9.

22. Delbarre A, Amor B, Bardoulat I, et al. Do intraarticular hyaluronic acid injections delay total knee replacement in patients with osteoarthritis-a Cox model analysis. PLoS One. 2017;12(11):e0187227.

23. Maheu E, Bannuru RR, Herrero-beaumont G, et al. Why we should definitely include intra-articular hyaluronic acid as a therapeutic option in the management of knee osteoarthritis: results of an extensive critical literature review. Semin Arthritis Rheumat. 2019. https://doi.org/10.1016/j. semarthrit.2018.06.002.

24. Maheu E, Rannou F, Reginster J. Efficacy and safety of hyaluronic acid in the management of osteoarthritis. Evid Real Life Sett Trials Surv. 2016;45:28-33. https:// doi.org/10.1016/j.semarthrit.2015.11.008.

25. Hempfling H. Intra-articular hyaluronic acid after knee arthroscopy: a two-year study. Knee Surg Sports Traumatol Arthrosc. 2007;15:537-46.

26. Zietz PM, Selesnick H. The use of hylan G-F 20 after knee arthroscopy in an active patient population with knee osteoarthritis. Arthroscopy. 2008;24: 416-22.

27. Chau JY, Chan WL, Woo SB, et al. Hyaluronic acid instillation following arthroscopic anterior cruciate ligament reconstruction: a double-blinded, randomised controlled study. J OrthopSurg (Hong Kong). 2012;20(2):162-5.

28. Product Information. Durolane ${ }^{\circledR}$ Instructions for use. Memphis: Smith and Nephew Inc.

29. Ayhan E, Kesmezacar H, Akgun I. Intraarticular injections (corticosteroid, hyaluronic acid, platelet rich plasma) for the knee osteoarthritis. World J Orthop. 2014;5:351-61.

30. Iannitti T, Lodi D, Palmieri B. Intra-articular injections for the treatment of osteoarthritis focus on the clinical use of hyaluronic acid. Drugs $\mathrm{R} D$. 2011;11(1):13-27.

31. Rosen J, Avram V, Fierlinger A, et al. Clinicians' perspectives on the use of intra-articular hyaluronic acid as a treatment for knee osteoarthritis: a North American, multidisciplinary survey. Clin Med Insights Arthritis Musculoskelet Disord. 2016;9:21-7.

32. Berenbaum F, Grifka J, Cazzaniga S, et al. A randomised, double-blind, controlled trial comparing two intra-articular hyaluronic acid preparations differing by their molecular weight in symptomatic knee osteoarthritis. Ann Rheum Dis. 2012;71(9): $1454-60$.

33. Shewale AR, Barnes CL, Fischbach LA, et al. Comparative effectiveness of low, moderate and high molecular weight hyaluronic acid injections in delaying time to knee surgery. J Arthroplasty. 2017;32(10):2952-7.

34. Kirchner M, Marshall D. A double-blind randomized controlled trial comparing alternate forms of high molecular weight hyaluronan for the treatment of osteoarthritis of the knee. Osteoarthritis Cartil. 2006;14:154-62.

35. Guo Y, Yang P, Liu L. Origin and efficacy of hyaluronan injections in knee osteoarthritis: randomized, double-blind trial. Med Sci Monit. 2018;24:4728-37.

36. Wen DY. Intra-articular hyaluronic acid injections for knee osteoarthritis. Am Fam Phys. 2000;62: 565-70.

37. Altman R, Hackel J, Niazi F, et al. Efficacy and safety of repeated courses of hyaluronic acid injections for knee osteoarthritis: a systematic review. Semin Arthritis Rheum. 2018;48(2):168-75.

38. Leighton R, Fitzpatrick J, Smith H, et al. Systematic clinical evidence review of NASHA (Durolane hyaluronic acid) for the treatment of knee osteoarthritis. Open Access Rheumatol. 2018;10:43-54.

39. Bannuru RR, Vaysbrot EE, McIntyre LF. Did the American Academy of Orthopaedic Surgeons osteoarthritis guidelines miss the mark? Arthroscopy. 2014;30:86-9. 